\title{
CIÊNCIAPNATURA 35产
}

\section{Desenvolvimento e validação de método para separação de isoflavonas em extrato seco de soja}

\author{
Development and validation of method for separation of isoflavones \\ in dry extract of soybean \\ Marcelo Ribani ${ }^{1}$, Carol H Collins², Carla Beatriz Grespan Bottoli ${ }^{3}$ \\ ${ }^{1}$ Instituto de Tecnologia do Paraná, Curitiba, Brasil \\ ${ }^{2}$ Departamento de Química Analítica, Instituto de Química - Unicamp, Campinas, Brasil \\ ${ }^{3}$ Departamento de Química Analítica, Instituto de Química - Unicamp, Campinas, Brasil
}

\begin{abstract}
Resumo
As isoflavonas são compostos fenólicos naturalmente encontrados em algumas plantas e são conhecidas como fitoestrogênios, compostos encontrados em alimentos derivados de plantas, primeiramente em produtos à base de soja. Neste trabalho foi desenvolvido um método de análise por Cromatografia Líquida de Alta Eficiência para separação e quantificação de isoflavonas presentes em extrato seco de soja. Para a validação do método, foram avaliados os parâmetros seletividade, precisão, exatidão, linearidade e faixa de trabalho, limite de detecção (LD), limite de quantificação (LQ), robustez e estabelecidos critérios de aceitabilidade para cada parâmetro. O método desenvolvido mostrou ser apropriado para determinação e quantificação de isoflavonas agliconas e glicosídicas presentes em extrato seco de soja.
\end{abstract}

Palavras-chave: Isoflavonas, extrato seco, soja, CLAE

\begin{abstract}
Isoflavones are phenolic compounds naturally found in some plants and are known as phytoestrogens, compounds found in plant-derived foods, primarily in products based on soybeans. In this work an analytical methodology for the High Performance Liquid Chromatographic separation and quantification of isoflavones present in dry soy extract was developed. In order to validate the method, parameters such as selectivity, precision, accuracy, linearity and working range, limit of detection (LOD), limit of quantification (LOQ) and robustness were evaluated. Acceptability criteria for each parameter were established. The methodology proved to be suitable for determination and quantification of the glycoside and aglycone isoflavones found in dry soy extract.
\end{abstract}

Keywords: Isoflavones, dry soy extract, soybean, HPLC 


\section{Introdução}

Entre os produtos agrícolas que alimentam a população mundial, a soja vem apresentando extraordinária expansão e ocupando uma posição de destaque. A plantação, crescimento e colheita de soja apresenta alta produtividade e uma fácil adaptação em quase todas as regiões do mundo.

O consumo de soja e produtos a base de soja está associado com benefícios à saúde, fato que criou considerável expectativa na comunidade dietética e nutricional. Isto ocorre porque esta leguminosa é um alimento que apresenta alto valor nutricional, tem uma composição química rica que inclui óleos, vitaminas e alguns sais minerais como cálcio e ferro, contém mais de $34 \%$ de proteína e é fonte de antioxidantes como as isoflavonas (SOUCI et al., 1994).

As isoflavonas são compostos fenólicos naturalmente encontrados em algumas plantas e são conhecidas como fitoestrogênios, compostos encontrados em alimentos derivados de plantas, primeiramente em produtos à base de soja. Suas estruturas são similares ao hormônio estrogênio, ligando-se aos receptores de estrogênio (PASCUAL-TERESA et al., 2006). Algumas isoflavonas (genistina, daidzina e glicitina) são encontradas apenas na soja, sendo consideradas antioxidantes naturais (FERRARI e DEMIATE, 2001). Com isso, o consumo de produtos à base de soja tem aumentado nos últimos anos, sendo uma alternativa à reposição hormonal, devido à sua atividade estrogênica e poucos efeitos colaterais. Acredita-se que as isoflavonas tenham uma ação potencial na prevenção de algumas doenças crônicas (IZUMI et al., 1997), como câncer relacionado à problemas hormonais (ADLERCREUTZ et al., 1991), osteoporoses (KNIGHT e EDEN, 1996), disfunções cardiovasculares (CLARKSON et al., 1995), câncer de colo (WATANABE e KOESSEL, 1993), câncer de próstata (SEVERSON et al., 1989) e ainda é indicada para diminuição dos sintomas ligados à menopausa (ANDERSON et al., 1999; KURZER, 2000).

As formas predominantes de isoflavonas em soja e em produtos não fermentados de soja são as formas glicosídicas (NAIN et al., 1974) e suas estruturas químicas podem ser visualizadas na figura 1 . Em produtos fermentados de soja, as formas predominantes são as agliconas. Após o consumo de produtos que contenham as isoflavonas glicosídicas, estas passam pelo metabolismo enzimático no intestino delgado para serem transformadas em agliconas (BOWEY et al., 2003), que é a forma da isoflavona mais biodisponível para o organismo (IZUMI et al., 2000).

Para determinação do teor de isoflavonas em grãos e outras matrizes de soja, uma das técnicas analíticas utilizadas é a cromatografia líquida de alta eficiência (HPLC) (COWARD et al., 1998; KLUMP et al., 2001; SETCHELL

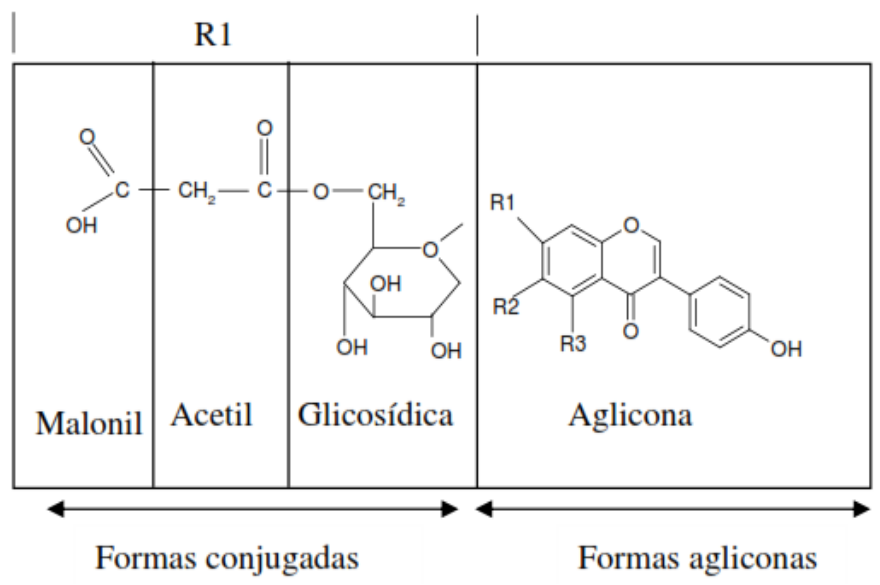

\begin{tabular}{lccc}
\hline Isoflavonas & R1 & R2 & R3 \\
\hline Genisteína & $\mathrm{OH}$ & $\mathrm{H}$ & $\mathrm{OH}$ \\
Daidzeína & $\mathrm{OH}$ & $\mathrm{H}$ & $\mathrm{H}$ \\
Gliciteína & $\mathrm{OH}$ & $\mathrm{OCH}_{3}$ & $\mathrm{H}$ \\
Genistina & $\mathrm{C}_{6} \mathrm{O}_{5} \mathrm{H}_{11}$ & $\mathrm{H}$ & $\mathrm{OH}$ \\
Daidzina & $\mathrm{C}_{6} \mathrm{O}_{5} \mathrm{H}_{11}$ & $\mathrm{H}$ & $\mathrm{H}$ \\
Glicitina & $\mathrm{C}_{6} \mathrm{O}_{5} \mathrm{H}_{11}$ & $\mathrm{OCH}_{3}$ & $\mathrm{H}$ \\
Acetil-genistina & $\mathrm{C}_{6} \mathrm{O}_{5} \mathrm{H}_{11}+\mathrm{COCH}_{3}$ & $\mathrm{H}$ & $\mathrm{OH}$ \\
Acetil-daidzina & $\mathrm{C}_{6} \mathrm{O}_{5} \mathrm{H}_{11}+\mathrm{COCH}_{3}$ & $\mathrm{H}$ & $\mathrm{H}$ \\
Acetil-glicitina & $\mathrm{C}_{6} \mathrm{O}_{5} \mathrm{H}_{11}+\mathrm{COCH}_{3}$ & $\mathrm{OCH}_{3}$ & $\mathrm{H}$ \\
Malonil-genistina & $\mathrm{C}_{6} \mathrm{O}_{5} \mathrm{H}_{11}+\mathrm{COCH}_{2} \mathrm{COOH}$ & $\mathrm{H}$ & $\mathrm{OH}$ \\
Malonil-daidzina & $\mathrm{C}_{6} \mathrm{O}_{5} \mathrm{H}_{11}+\mathrm{COCH}_{2} \mathrm{COOH}$ & $\mathrm{H}$ & $\mathrm{H}$ \\
Malonil-glicitina & $\mathrm{C}_{6} \mathrm{O}_{5} \mathrm{H}_{11}+\mathrm{COCH}_{2} \mathrm{COOH}$ & $\mathrm{OCH}_{3}$ & $\mathrm{H}$ \\
\hline
\end{tabular}

Figura 1: Estrutura química das isoflavonas encontradas em grãos de soja. 
et al., 2001; WANG e MURPHY, 1994; WISEMAN et al., 2002) que, após a extração das isoflavonas, permite sua separação e quantificação.

A maioria dos métodos que empregam a cromatografia líquida utilizam o modo de eluição por gradiente para separação e utilizam, como fase móvel, misturas de metanol:água ou acetonitrila:água e ácido trifluoroacético ou ácido acético glacial como modificador (ELDRIDGE, 1982; MURPHY, 1981; FARMAKALIDIS e MURPHY, 1984). Entretanto, estes métodos não têm sido bem sucedidos na resolução das 12 isoflavonas simultaneamente, mesmo utilizando programação por gradiente. WANG e MURPHY, 1994, relataram uma condição reprodutível que permitiu uma boa separação para as 12 isoflavonas, porém com um tempo de 90 min de análise total. Outros métodos também foram desenvolvidos por MURPHY et al., 1997; FRANKE et al., 1999 e SHAO et al., 2011, embora com tempos de corrida longos e resolução insuficiente.

Métodos mais rápidos e mais simples foram também desenvolvidos e utilizados para a análise simultânea de 12 isoflavonas empregando uma fase móvel composta de acetonitrila:água com gradiente binário. Entretanto estes métodos apresentaram sobreposição do padrão interno com a genistina (HSEIH et al., 2004; KAO e CHEN, 2002; SONG E MURPHY, 1998).

CÉSAR et al., 2006, estabeleceram um método usando condição isocrática para separação e quantificação das isoflavonas na forma aglicona (daidzeína, gliciteína e genisteína) para o extrato seco de soja. Este método utilizou hidrólise ácida para transformar todas as formas glicosídicas na forma aglicona, permitindo separar três isoflavonas na forma aglicona das impurezas provenientes do extrato seco.

Neste trabalho foi desenvolvido um método analítico por Cromatografia Líquida de Alta Eficiência para separação e quantificação de isoflavonas presentes em extrato seco de soja.

Neste método foram preservadas tanto as isoflavonas na forma glicosídica, quanto as isoflavonas na forma aglicona presentes no extrato seco para confirmar os teores de cada isoflavona individualmente.

Para a validação do método, foram avaliados os parâmetros seletividade, precisão, exatidão, linearidade e faixa de trabalho, limite de detecção (LD), limite de quantificação (LQ), robustez e estabelecidos critérios de aceitabilidade para cada parâmetro.

\section{Material e Método}

\subsection{Equipamento analítico}

O sistema cromatográfico analítico utilizado no desenvolvimento da separação e na validação foi um cromatógrafo Merck Hitachi LaChrom, composto de uma bomba quaternária L-7100, um injetor automático L-7250, um detector UV-Vis com varredura espectral L-7455, um módulo de aquecimento de colunas L-7300 e o software Merck HSM, versão 4.1, foi usado no tratamento dos dados. A coluna usada foi a Nova-Pak C-18 (150 mm x 3,9 mm d.i.), com partículas de $4 \mu \mathrm{m}$, Waters. A coluna foi mantida na temperatura de $35^{\circ} \mathrm{C}$. A fase móvel foi um gradiente de água acidificada com $0,1 \%$ ácido acético glacial pH 3,5 (Solução A) e acetonitrila acidificada com 0,1\% ácido acético glacial (Solução B). A programação do gradiente da fase móvel foi: $14 \%$ of B por 8 min; 14 $\%$ até $21 \%$ B em 4 min; $21 \%$ de B por 3 min; $21 \%$ até 29 \% B em 5 min; $29 \%$ para $40 \%$ B em 3 min; $40 \%$ até $50 \%$ B em 2 min; $50 \%$ de B por 5 min; $50 \%$ até $14 \%$ B em 5 min; $14 \%$ B por 3 min. O volume de injeção foi de $10 \mathrm{~mL}$ e o sistema de detecção utilizado foi UV-vis com varredura espectral de 200 a $400 \mathrm{~nm}$, monitorado em $254 \mathrm{~nm}$. A vazão da fase móvel foi mantida constante por todo o tempo da análise a 1,0 $\mathrm{mL} \mathrm{min}^{-1}$.

\subsection{Padrões de isoflavonas}

Os padrões de referência de isoflavonas utilizados foram: daidzeína 99,9\%, gliciteína 96,3 \%, genisteína $99,6 \%$ (isoflavonas na forma aglicona), daidzina 99,2\%, glicitina $87,4 \%$ e genistina $98,2 \%$ (isoflavonas na forma glicosídica) obtidos da Chromadex, certificados internacionalmente pela USP (United Stated Pharmacopeia) e FDA (Food and Drug Administration). Os padrões foram dissolvidos em solução metanol:água (80:20 v/v).

\subsection{Amostras de extrato seco de soja}

As amostras de extrato seco de soja padronizado com $40 \%(\mathrm{~m} / \mathrm{m})$ de isoflavonas, foram fornecidas pelo laboratório botânico Herbarium (Curitiba, Paraná) e em todo trabalho foi empregado o extrato seco proveniente de um mesmo lote.

Foram pesados $50 \mathrm{mg}$ de amostras de extrato seco de soja a $40 \%$ em balão volumétrico de $50 \mathrm{~mL}$ e adicionado solução metanol:água $(80: 20 \mathrm{v} / \mathrm{v})$, obtendo assim concentração de $1 \mathrm{mg} \mathrm{mL}^{-1}$. As amostras foram colocadas em banho ultra-sônico por 20 minutos, agitadas vigorosamente e filtradas em membrana de 0,45 $\mu \mathrm{m}$ de porosidade, antes de serem injetadas no sistema cromatográfico, utilizando as condições cromatográficas otimizadas. Foram determinadas as concentrações de cada uma das isoflavonas nesta amostra, em triplicata e expressos como a média do teor de cada isoflavona.

\subsection{Validação do método}

\subsubsection{Seletividade}

Para avaliar e comprovar a seletividade na separação cromatográfica para cada um dos compostos, foram preparadas soluções padrão contendo daidzina $(4 \mu \mathrm{g}$

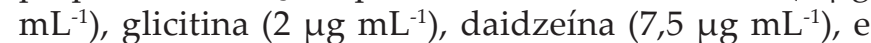
genisteína (30 $\left.\mu \mathrm{g} \mathrm{mL}^{-1}\right)$ em solução metanol : água (80:20 
v/v). A solução da amostra de extrato seco de soja foi preparada na concentração de $1 \mathrm{mg} \mathrm{mL}^{-1}$, fortificada com os padrões. Os cromatogramas obtidos dos padrões e da amostra fortificada foram comparados pelo tempo de retenção de cada composto. Também foram monitorados os espectros na faixa de 200 a $400 \mathrm{~nm}$, tanto dos padrões quanto da amostra, assim como foi avaliada a pureza espectral de todos os compostos a fim de confirmar a seletividade do método na separação das isoflavonas frente aos interferentes do extrato.

\subsubsection{Linearidade e faixa de aplicação}

A linearidade foi verificada primeiramente pelo coeficiente de correlação linear, r, resultante da linha de regressão de cinco concentrações diferentes, para cada composto, individualmente e, confirmada pelo método do gráfico de linearidade. As concentrações utilizadas foram: $1,2,4,10$ e $15 \mu \mathrm{g} \mathrm{mL}^{-1}$ para daidzina; $1,1,5,2,4$ e $10 \mu \mathrm{g} \mathrm{mL}{ }^{-1}$ para glicitina; $1,5,7,5,10$ e $15 \mu \mathrm{g} \mathrm{mL}^{-1}$ para daidzeína; e 10, 20, 30, 40 e $50 \mu \mathrm{g} \mathrm{mL}^{-1}$ para genisteína, dissolvidos em solução metanol: água (80:20 v/v).

\subsubsection{Limite de deteç̧ão (LD) e limite de quantifi- cação (LQ)}

Os limites de detecção (LD) e de quantificação (LQ) foram calculados através do método baseado em parâmetros da curva analítica (RIBANI et al., 2004 e 2007), conforme as equações 1 e 2 abaixo.

$$
\begin{array}{ll}
\mathrm{LD}=3,3 \times \frac{s}{\mathrm{~S}} & \text { (equação 1) } \\
\mathrm{LQ}=10 \times \frac{s}{\mathrm{~S}} & \text { (equação 2) }
\end{array}
$$

onde:

$\mathrm{s}=$ estimativa do desvio padrão do coeficiente linear da equação.

$\mathrm{S}=$ inclinação ou coeficiente angular da curva analítica.

Posteriormente foram injetadas soluções nas concentrações determinadas para confirmação visual dos picos de cada um dos compostos.

\subsubsection{Precisão}

A precisão representa a dispersão de resultados entre ensaios independentes, repetidos de uma mesma amostra, amostras semelhantes ou padrões, sob condições definidas.
Foram preparadas 8 (oito) soluções da amostra de extrato seco de soja padronizado, na concentração de 1 $\mathrm{mg} \mathrm{mL}^{-1} \mathrm{em}$ solução metanol: água $(80: 20 \mathrm{v} / \mathrm{v})$, deixados por 20 minutos em banho ultra-som e realizaram-se 3 (três) injeções de cada uma destas soluções. A precisão dos resultados repetidos para a mesma amostra, para cada isoflavona, foi avaliada conforme a norma ISO 5725, 1994.

Foram calculadas e expressas as médias, as estimativas de desvios padrões, coeficientes de variação, o limite de repetitividade em termos relativos e absolutos, para cada isoflavona.

Para o cálculo do limite de repetitividade $\left(\mathrm{r}^{\prime}\right)$ da precisão (IN METRO, 2003), utilizou-se a equação 3 e aplicou-a para cada isoflavona individualmente:

$\mathrm{r}^{\prime}=2,8 \times \mathrm{sr}$ (equação 3)

onde:

sr = estimativa do desvio padrão da concentração das 8 (oito) soluções amostra preparadas.

O limite de repetitividade encontrado deve ser menor que $5 \%$ para cada uma das isoflavonas presentes no extrato seco.

\subsubsection{Exatidão}

Como não havia material de referência certificado (CRM) de extrato seco de soja para comparação, foram utilizados padrões de referência das isoflavonas adicionados nas amostras e dissolvidos em solução metanol:água (80:20 v/v) nas concentrações de: 2 , 4 e 10 $\mu \mathrm{g} \mathrm{mL}{ }^{-1}$ para daidzina; 1,5 , 2 e $4 \mu \mathrm{g} \mathrm{mL}^{-1}$ para glicitina; 5, 7,5 e $10 \mu \mathrm{g} \mathrm{mL}^{-1}$ para daidzeína; 20, 30 e $40 \mu \mathrm{g} \mathrm{mL}^{-1}$ para genisteína. Desta maneira, a exatidão foi avaliada pela recuperação $(R)$ em triplicata, em cada nível de concentração e os resultados foram expressos em porcentagem, conforme equação 4 .

Para verificar a exatidão, utilizou-se o teste $t$ de Student (MILLER E MILLER, 2002), no qual foi feita a

$$
\mathrm{R}(\%)=\frac{\text { média do valor obtido }}{\text { média do valor adicionado }} \times 100
$$

comparação da média experimental ( $\bar{x})$, valor obtido pela média aritmética de nove resultados experimentais em termos de porcentagem, com o valor verdadeiro $(\mu$ $=100 \%$ ) obtido pela quantidade adicionada, conforme a equação 5 :

$$
t_{s}=(\bar{x}-\mu) \sqrt{n} / s
$$

(equação 5) 
Os resultados encontrados foram expressos em porcentagem e avaliados pelo teste $t$ de Student. Os resultados calculados para $t$, foram comparados com os valores $t$ tabelados para $95 \%$ de confiabilidade.

\subsubsection{Robustez}

A robustez de um método mede a sensibilidade que este apresenta face à pequenas variações. Diz-se que um método é robusto quando ele não é afetado por uma pequena e deliberada modificação em seus parâmetros.

Verificou-se a robustez do método para determinação de isoflavonas em extrato seco de soja por cromatografia líquida de alta eficiência, aplicando o teste de Youden (IN METRO, 2003; YOUDEN E STEINER, 1975).

As variações no método foram:

a) concentração de solvente orgânico para extração;

b) tempo de extração em ultra-som;

c) temperatura da solução durante a extração;

Realizou-se 8 (oito) ensaios separados para determinar os efeitos da variação de cada um dos parâmetros.

Elaborou-se a tabela 1 com os fatores nominais do método, representados pelo sinal (+) e as respectivas variações a serem testadas, representadas pelo sinal (-). Os resultados da combinação em porcentagem entre estes ensaios foram representados com letras minúsculas.

Tabela 1: Combinação ensaiada para avaliação da robustez para cada isoflavona.

\begin{tabular}{c|c|c|c|c}
\hline \multirow{2}{*}{$\begin{array}{c}\text { Combinação } \\
\text { ensaiada }\end{array}$} & $\begin{array}{c}\text { Concentração } \\
\text { MeOH:H2O } \\
(\mathrm{v} / \mathrm{v})\end{array}$ & $\begin{array}{c}\text { Tempo de } \\
\text { ultrassom } \\
(\mathrm{min})\end{array}$ & $\mathrm{T}\left({ }^{\circ} \mathrm{C}\right)$ & $\begin{array}{c}\text { Identificação } \\
\text { dos } \\
\text { resultados }\end{array}$ \\
\hline 1 & $80: 20(+)$ & $20(+)$ & $55(+)$ & $\mathrm{a}$ \\
\hline 2 & $80: 20(+)$ & $20(+)$ & $50(-)$ & $\mathrm{b}$ \\
\hline 3 & $80: 20(+)$ & $10(-)$ & $55(+)$ & $\mathrm{c}$ \\
\hline 4 & $80: 20(+)$ & $10(-)$ & $50(-)$ & $\mathrm{d}$ \\
\hline 5 & $82: 18(-)$ & $20(+)$ & $55(+)$ & $\mathrm{e}$ \\
\hline 6 & $82: 18(-)$ & $20(+)$ & $50(-)$ & $\mathrm{f}$ \\
\hline 7 & $82: 18(-)$ & $10(-)$ & $55(+)$ & $\mathrm{g}$ \\
\hline 8 & $82: 18(-)$ & $10(-)$ & $55(-)$ & $\mathrm{h}$ \\
\hline
\end{tabular}

Aplicando o teste de Youden, o cálculo do efeito da significância foi realizado conforme segue abaixo:

Efeito concentração metanol: água =

$$
\frac{a+b+c+d}{4}-\frac{e+f+g+h}{4}
$$

(equação 6)

Efeito tempo de ultrassom $=$

$$
\frac{a+b+e+f}{4}-\frac{c+d+g+h}{4}
$$

Efeito temperatura $=$

$\frac{a+c+e+g}{4}-\frac{b+d+f+h}{4}$

(equação 8)

Este cálculo indica se houve ou não efeito significativo nos fatores, para um critério de significância de duas vezes o $C V$ em relação ao resultado da precisão do método para todos os efeitos calculados, para cada uma das isoflavonas. Os resultados destas variações foram comparados com os resultados da precisão do método.

\section{Resultados e Discussão}

Várias condições foram avaliadas até a obtenção da melhor separação cromatográfica de todas as isoflavonas. As variações foram feitas na fase móvel iniciando as separações com um programa de gradiente o qual utilizava metanol e água acidificada como fase móvel e, posteriormente, modificando para acetonitrila e água acidificada. A fase móvel contendo metanol deixava os picos muito largos e por este motivo, foi substituída pela acetonitrila. A melhor condição para separação das isoflavonas está apresentada na figura 2.

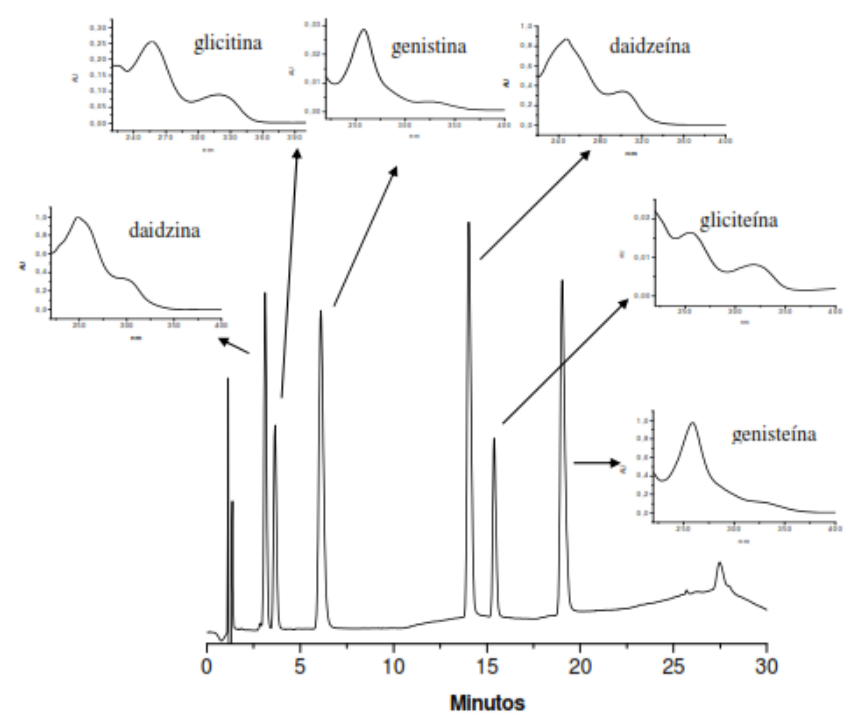

Figura 2: Separação cromatográfica da mistura de padrões de isoflavonas com os espectros de cada isoflavona obtido com o detector de arranjo de diodos. Concentração das isoflavonas: $100 \mu \mathrm{g} \mathrm{mL}^{-1}$.

Condições cromatográficas: Coluna analítica: Nova Pak Waters (150 x 3,9 mm) C-18, partículas esféricas de $4 \mathrm{~mm}$; Fase móvel: água + 0,1\% ácido acético glacial, pH 3,5 (A); acetonitrila $+0,1 \%$ ácido acético glacial (B). Programa de gradiente: $21 \%$ de B por 8 min; $21 \%$ até $27 \% \mathrm{~B}$ em 4 min; $27 \%$ de B por 3 min; 
$27 \%$ até $31 \%$ B em 5 min; $31 \%$ para $40 \%$ B em 3 min; $40 \%$ até $50 \% \mathrm{~B}$ em $2 \mathrm{~min} ; 50 \%$ de B por $5 \mathrm{~min}$; $50 \%$ até $21 \%$ B em 5 min; $21 \%$ B por 3 min. Volume de injeção: $10 \mathrm{~mL}$. Detecção: UV-vis com varredura espectral de 200 a $400 \mathrm{~nm}$, monitorado em $254 \mathrm{~nm}$. Vazão: $1,0 \mathrm{~mL} \mathrm{~min}{ }^{-1}$. Temperatura da coluna: $35{ }^{\circ} \mathrm{C}$.

Após obter a melhor separação para as isoflavonas, o método de análise foi validado e, em seguida foram calculados os teores de isoflavona em cada uma de suas formas.

Como neste lote de extrato seco continha somente 4 isoflavonas, duas na forma aglicona (daidzina, glicitina) e duas na forma glicosídica (daidzeína e genisteína), os resultados da validação serão apresentados somente para estes compostos.

A seletividade foi confirmada pelo tempo de retenção dos compostos e pela comparação e pureza espectral, numa faixa de concentração similar entre os padrões de isoflavonas e as amostras de extrato seco fortificadas com os padrões de isoflavonas.

Pode-se observar na figura 3 o cromatograma da amostra de extrato seco com os respectivos espectros das isoflavonas: daidzina, glicitina, daidzeína e genisteína.

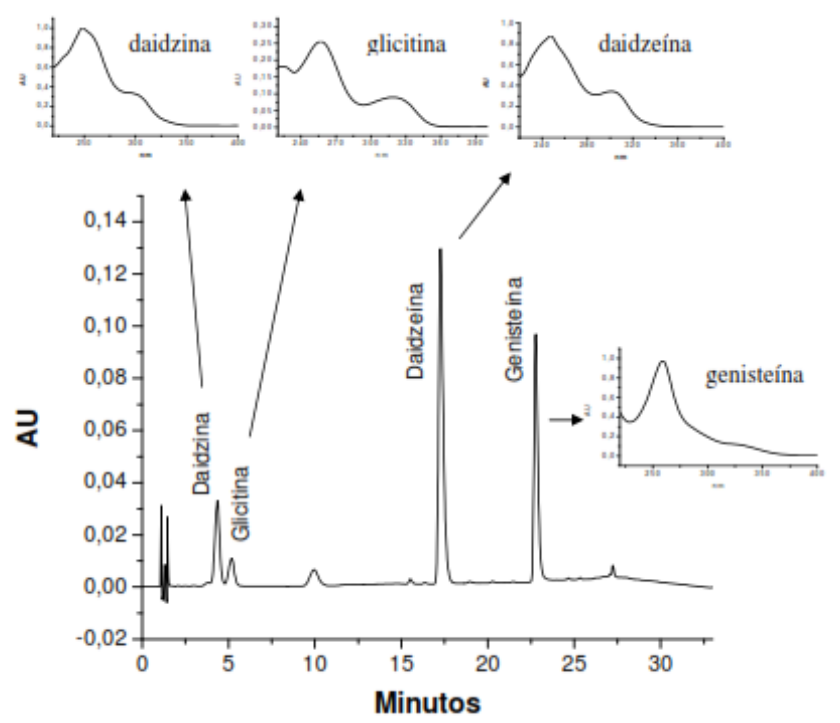

Figura 3: Cromatograma da amostra de extrato seco de soja com os espectros de cada isoflavona obtidos com o detector por arranjo de diodos e comparados com os espectros dos padrões.

Condições cromatográficas: Ver em Material e Método.

Na construção da curva analítica observou-se que não houve interferência da matriz na quantificação das isoflavonas na faixa de concentração estudada, devido aos coeficientes angulares serem similares entre a curva analítica somente com o padrão dissolvido na fase móvel e a curva analítica na matriz, permitindo assim o uso dos padrões preparados em solução metanol:água (80:20 v/v).

As concentrações utilizadas nas curvas analíticas foram $1,2,4,10$ e $15 \mu \mathrm{g} \mathrm{mL}^{-1}$ para daidzina; $1,1,5,2,4$ e $10 \mu \mathrm{g} \mathrm{mL}^{-1}$ para glicitina; $1,5,7,5,10$ e $15 \mu \mathrm{g} \mathrm{mL}^{-1}$ para daidzeína; e 10, 20, 30, 40 e $50 \mu \mathrm{g} \mathrm{mL}^{-1}$ para genisteína, dissolvidos em solução metanol:água $(80: 20 \mathrm{v} / \mathrm{v})$.

A linearidade foi confirmada pelo método do gráfico de linearidade (HUBER, 1998; CASSIDY e JANOSKI, 1992; RIBANI et al., 2007). Este método consiste na construção de um gráfico relacionando a área por concentração (eixo y) e log da concentração (eixo x). Estabeleceu-se o coeficiente angular como a reta central (paralela ao eixo X) do gráfico e definiu-se $15 \%$ acima e $15 \%$ abaixo deste valor, como o intervalo de confiabilidade, para confirmar a linearidade dos pontos no intervalo de concentração. Este gráfico permite visualizar como os pontos da curva analítica se comportam nesta faixa de concentração. Como nenhum ponto ficou fora do intervalo de confiança, a faixa de concentração foi definida como intervalo linear.

Os parâmetros da curva analítica para as isoflavonas estão apresentados na tabela 2.

Tabela 2: Parâmetros das curvas analíticas para cada isoflavona.

\begin{tabular}{c|c|c|c|c}
\hline & Daidzina & Glicitina & Daidzeína & Genisteína \\
\hline $\begin{array}{c}\text { Faixa de } \\
\text { aplicação } \\
\mu \mathrm{g} \mathrm{mL} \mathrm{L}^{-1}\end{array}$ & $1-10$ & $1-15$ & $1-10$ & $10-50$ \\
\hline $\begin{array}{c}\text { Coeficiente } \\
\text { correlação } \\
\mathrm{r}\end{array}$ & 0,9998 & 0,9996 & 0,9997 & 0,9996 \\
\hline $\begin{array}{c}\text { Coeficiente } \\
\text { angular }\end{array}$ & 36595 & 32673 & 42670 & 55189 \\
\hline $\begin{array}{c}\text { Coeficiente } \\
\text { linear }\end{array}$ & -1299 & 405 & -4840 & -23243 \\
\hline $\begin{array}{c}\text { Desvio padrão } \\
\text { do coeficiente } \\
\text { linear }\end{array}$ & 1352 & 1091 & 2440 & 12734 \\
\hline $\begin{array}{c}\text { Desvio padrão } \\
\text { da linha de } \\
\text { regressão }\end{array}$ & 3347 & 2818 & 4930 & 21029 \\
\hline
\end{tabular}

Para o cálculo de LD e de LQ, foram utilizados os parâmetros das curvas analíticas, de acordo com as equações 1 e 2 . A tabela 3 apresenta os valores de LD e de LQ para cada isoflavona.

Os valores de LQ foram também confirmados visualmente, a partir da injeção de soluções nas concentrações obtidas pelo método da curva analítica.

As concentrações de isoflavonas presentes no extrato seco foram avaliadas em seis amostras de um mesmo lote e foram calculados as médias, as estimativas dos desvios padrões, os coeficientes de variação e os limites de repetitividade. Os resultados analíticos e estatísticos para cada isoflavona estão na tabela 4 . 
Tabela 3: Valores de LD e LQ determinados pelo método baseado nos parâmetros da curva analítica.

\begin{tabular}{l|cccc}
\hline Isoflavonas & $\begin{array}{c}\text { LD do } \\
\text { instrumento } \\
(\mu \mathrm{g} / \mathrm{mL})\end{array}$ & $\begin{array}{c}\text { LQ do } \\
\text { instrumento } \\
(\mu \mathrm{g} / \mathrm{mL})\end{array}$ & $\begin{array}{c}\text { LD do } \\
\text { método } \\
(\mathrm{g} / 100 \mathrm{~g})\end{array}$ & $\begin{array}{c}\text { LQ do } \\
\text { método } \\
(\mathrm{g} / 100 \mathrm{~g})\end{array}$ \\
\hline Daidzina & 0,1 & 0,4 & 0,1 & 0,4 \\
Daidzeína & 0,2 & 0,6 & 0,2 & 0,6 \\
Glicitina & 0,1 & 0,3 & 0,1 & 0,3 \\
Genisteína & 0,1 & 0,3 & 0,1 & 0,3 \\
\hline
\end{tabular}

Tabela 4: Parâmetros de precisão no nível de repetitividade para os teores de isoflavonas contidas nas amostras de extrato seco de soja.

\begin{tabular}{c|cccc}
\hline $\begin{array}{c}\text { Amostras } \\
(\mathrm{n}=8)\end{array}$ & $\begin{array}{c}\text { Daidzina } \\
\mathrm{g} / 100 \mathrm{~g}\end{array}$ & $\begin{array}{r}\text { Daidzeína } \\
\mathrm{g} / 100 \mathrm{~g}\end{array}$ & $\begin{array}{c}\text { Glicitina } \\
\mathrm{g} / 100 \mathrm{~g}\end{array}$ & $\begin{array}{c}\text { Genisteína } \\
\mathrm{g} / 100 \mathrm{~g}\end{array}$ \\
\hline Média, $\bar{x}$ & 7,9 & 24,4 & 3,2 & 10,5 \\
\hline $\begin{array}{c}\text { Estimativa do } \\
\text { desvio padrão Sr }\end{array}$ & 0,1 & 0,2 & 0,1 & 0,1 \\
\hline $\begin{array}{c}\text { Coeficiente de } \\
\text { Variação - CV (\%) }\end{array}$ & 1,4 & 1,0 & 1,5 & 1,3 \\
\hline $\begin{array}{c}\text { Limite de } \\
\text { repetitividade - } \mathrm{r}^{\prime}\end{array}$ & 0,3 & 0,7 & 0,1 & 0,4 \\
\hline $\begin{array}{c}\text { Limite de } \\
\text { repetitividade - } \\
\text { r' cv (\%) }\end{array}$ & 3,9 & 2,7 & 4,1 & 3,7 \\
\hline
\end{tabular}

Tabela 5: Valores da recuperação das isoflavonas, do CV e do teste ts de Student.

\begin{tabular}{c|cccc}
\hline & $\begin{array}{c}\text { Daidzina } \\
\%\end{array}$ & $\begin{array}{c}\text { Daidzeína } \\
\%\end{array}$ & $\begin{array}{c}\text { Glicitina } \\
\%\end{array}$ & $\begin{array}{c}\text { Genisteína } \\
\%\end{array}$ \\
\hline $\begin{array}{c}\text { Recuperação } \\
(\mathrm{n}=9)\end{array}$ & 100,9 & 100,1 & 100,7 & 98,9 \\
\hline $\begin{array}{c}\text { Coeficiente de } \\
\text { variação, } \\
\text { CV (\%) }\end{array}$ & 1,5 & 1,6 & 1,5 & 1,6 \\
\hline$t_{\text {s calculado }}$ & 1,8 & 0,2 & 1,2 & 2,0 \\
\hline$t_{s}$ tabelado (95\%) & 2,262 & 2,262 & 2,262 & 2,262 \\
\hline
\end{tabular}

A partir da estimativa do desvio padrão, pode-se calcular o limite de repetitividade $\left(\mathrm{r}^{\prime}\right)$ que permite ao analista decidir se a diferença entre análises realizadas em duplicatas de uma amostra, determinada sob condições de repetitividade, é significante. Os valores do limite de repetitividade permitem a avaliação de amostras rotineiras e a verificação de diferenças significativas num nível de $95 \%$ de confiabilidade.

O método demonstrou ser preciso para uma distribuição normal de ts de Student, de acordo com os parâmetros apresentados na tabela 4 . Os valores de $\mathrm{r}^{\prime}$ indicam que, em análises rotineiras realizadas em duplicatas de amostras, a amplitude entre os resultados não poderá ser maior que este valor, para que o processo analítico esteja sob controle.

As variações apresentadas entre os resultados para cada isoflavona, apresentaram uma variação inferior a $5 \%$, indicando uma precisão adequada ao método.

A tabela 5 apresenta os valores de recuperação e os valores calculados para ts de Student, conforme equação 5. O critério para verificar a exatidão do método foi a comparação entre o valor experimental determinado e o valor tabelado para o ts de Student para seis determinações feitas no mesmo dia e dentro de um intervalo curto de tempo utilizando resultados de recuperação.

Os valores calculados para ts de Student são menores que os valores tabelados, demonstrando que o método apresenta exatidão dentro de um intervalo de confiança de $95 \%$.

A robustez foi avaliada pelo teste de Youden. Os parâmetros avaliados foram a concentração de metanol na extração, o tempo em que as amostras foram deixadas 
Tabela 6: Resultados expressos em \% de recuperação das combinações ensaiadas na avaliação da robustez para cada isoflavona.

\begin{tabular}{c|l|l|l|l}
\hline \multirow{2}{*}{$\begin{array}{c}\text { Combinação } \\
\text { ensaiada }\end{array}$} & \multicolumn{4}{|c}{ Isoflavonas } \\
\cline { 2 - 5 } & Daidzina & Glicitina & Daidzeína & Genisteína \\
\hline $\mathrm{a}$ & 100,7 & 100,3 & 100,1 & 99,4 \\
\hline $\mathrm{b}$ & 99,7 & 99,2 & 99,5 & 100,2 \\
\hline $\mathrm{c}$ & 98,9 & 100,1 & 99,8 & 100,4 \\
\hline $\mathrm{d}$ & 98,8 & 100,2 & 99,1 & 100,1 \\
\hline $\mathrm{e}$ & 98,8 & 99,9 & 100,2 & 99,9 \\
\hline $\mathrm{f}$ & 98,8 & 99,1 & 100,5 & 99,6 \\
\hline $\mathrm{g}$ & 99,1 & 100,2 & 100,1 & 99,6 \\
\hline $\mathrm{h}$ & 100,7 & 100,1 & 98,9 & 99,8 \\
\hline
\end{tabular}

Tabela 7: Comparação entre os efeitos avaliados no teste de Youden com a precisão do método.

\begin{tabular}{c|c|ccc|}
\hline & & \multicolumn{3}{|c|}{ Efeito dos parâmetros } \\
\hline & $\begin{array}{c}\text { CV do } \\
\text { método }(\%)^{*}\end{array}$ & $\begin{array}{c}\text { Concentração } \\
\text { de MeOH }\end{array}$ & $\begin{array}{c}\text { Tempo } \\
\text { ultrassom }\end{array}$ & Temperatura \\
\hline Daidzina & 1,4 & 1,4 & 0,5 & 1,4 \\
Glicitina & 1,5 & 1,5 & 2,1 & 1,5 \\
Daidzeína & 1,0 & 1,0 & 2,4 & 1,0 \\
Genisteína & 1,3 & 1,3 & 0,8 & 1,3 \\
\hline
\end{tabular}

* Valores obtidos na avaliação da precisão do método (tabela 4)

Tabela 8: Teores de isoflavonas presentes no extrato seco de soja padronizado.

\begin{tabular}{c|cc}
\hline Isoflavona & $\mathrm{g} / 100 \mathrm{~g}$ & Incerteza \\
\hline Daidzeína & 24,4 & $\pm 0,2$ \\
Daidzina & 7,9 & $\pm 0,1$ \\
Glicitina & 3,2 & $\pm 0,1$ \\
Genisteína & 10,5 & $\pm 0,1$ \\
\hline
\end{tabular}

em banho ultrassom e a temperatura durante a extração.

Foram realizados oito ensaios conforme a combinação dos parâmetros indicada na tabela 1 .

Os resultados, em termos de porcentagem recuperada, de cada uma das isoflavonas, em cada um dos ensaios, pode ser visto na tabela 6 e os $C V(\%)$ resultantes de cada um dos ensaios calculados pelas equações 6,7 e 8 estão na tabela 7. Os valores dos efeitos foram comparados com os valores de $\mathrm{CV}$ encontrados na precisão do método (tabela 4), sendo que os efeitos devem apresentar valores menores que duas vezes os valores de $\mathrm{CV}$ para não mostrarem efeito significativo na precisão do método.

Como os efeitos estudados não apresentaram valores maiores que dois $\mathrm{CV}$, nenhum dos parâmetros avaliados mostrou efeito significativo, indicando que o método pode ser considerado robusto frente a estas variações experimentais.

O método validado foi utilizado para determinação dos teores de cada uma das isoflavonas presentes no lote de extrato seco utilizado neste estudo. Os resultados e a incerteza associada, calculados pelo intervalo de confiança, estão descritos na tabela 8 .

\section{Conclusões}

Os teores e a composição de isoflavonas em extratos secos podem variar consideravelmente dependendo do lote e do fornecedor. Normalmente estes extratos, encontrados comercialmente, são padronizados para conter um total de $40 \%(\mathrm{~m} / \mathrm{m})$ de isoflavonas. No entanto, os teores individuais de isoflavonas não são controlados e podem variar devido a uma série de influências como as condições climáticas, a variedade da soja, o período da colheita e a localização geográfica.

O método desenvolvido mostrou ser apropriado para determinação e quantificação de isoflavonas agliconas e glicosídicas presentes em extrato seco de soja. 


\section{Agradecimentos}

Os autores agradecem o laboratório botânico Herbarium (Curitiba, Paraná) pelo fornecimento dos extratos secos de soja e o CNPq pelas bolsas de produtividade em pesquisa.

\section{Referências}

ADLERCREUTZ, H.; HONJO, H.; HIGASHI, A.; FOTSIS, T.; HAMALAINEN, E.; HASEGAWA, T.; OKADA, H. Urinary excretion of lignans and isoflavonoid phytoestrogens in Japanese men and women consuming a traditional diet. American Journal of Clinical Nutrition, v. 54, p. 1093-1100, 1991.

ANDERSON, J.J.B.; ANTHONY, M.S.; CLINE, J. M.; WASHBURN, S.A.; GARNER, S.C. Health potential of soy isoflavones for menopausal women. Public Health Nutrition, v.2, p. 489-504, 1999.

BOWEY, E.; ADLERCREUTZ, H.; ROWLAND, I. Metabolism of isoflavones and lignans by the gut microflora: study in germ-free and human flora associated rats. Food Chemistry Toxicology, v.41, p. 631-636, 2003.

CASSIDY, R.; JANOSKI, M. Is your calibration curve linear?. LC-GC, v.10, p. 692-696, 1992.

CÉSAR, I.C.; BRAGA, F.C.; SOARES, C.D.V.; NUNAN, E.A.; PIANETTI, G.A.; CONDESSA, F.A.; BARBOSA, T.A.F.; CAMPOS, L.M.M. Development and validation of a RP-HPLC method for quatification of isoflavoneaglycones in hydrolyzed soy dry extracts. Journal of Chromatography B, v.836, p. 74-78, 2006.

CLARKSON, T. B.; ANTHONY, M. S.; HUGHES, C. L. Estrogenic soybean isoflavones and chronic disease Risks and benefits. Trends in Endocrinology \& Metabolism, v. 6, p. 11-16, 1995.

COWARD, L.; SMITH, M.; KIRK, M.; BARNES, S. Chemical modification of isoflavones in soyfoods during cooking and processing. The America Journal of Clinical Nutrition, v. 68, p.1486-1491, 1998.

ELDRIDGE, A. C. High-performance liquid chromatography separation of soybean isoflavones and their glucosides. Journal of Chromatography A, v.234, p. 494-496, 1982.
FARMAKALIDIS, E.; MURPHY, P. A. Semipreparative high performanceliquid chromatographic isolation of soybean isoflavones. Journal of Chromatography A, v.295, p. 510, 1984.

FERRARI, R.A.; DEMIATE, I.M. Soybean isoflavones - a short review. Biological Health Science, v. 7, p. 39-46, 2001.

HSEIH, H. C.; KAO, T. H.;CHEN, B. H. A fast HPLC method for analysis of isoflavones in soybean. Journal of Liquid Chromatography \& Related Technologies, v. 27, p.315-324, 2004.

HUBER, L. Validation of analytical methods: review and strategy. LC-GC International, v. 11, p. 96-105, 1998.

International Standards Organization: ISO 5725-6: Accuracy (trueness and precision) of Measurement Methods and Results - Use in Pratice of Accuracy Values, International Organization for Standardization. Geneva, 1994.

Instituto Nacional de Metrologia, Normalização e Qualidade Industrial (INMETRO): DOQCGCRE-008: Orientações sobre Validação de Métodos de Ensaios Químicos. Brasília, 2003.

IZUMI, T.; NASU, A.; KATAOKA, S.; TOKUTAKE, S.; OBATA, A.; TOBE, K. An efficient preparation of acetyl isoflavoneglucoside. Chemical \& Pharmaceutical Bulletin, v. 45, n.10, p. 1593-1595, 1997.

IZUMI, T.; PISKULA, M.K.; OSAWA, O.; OBATA, A.; TOBE, K.; SAITO, M.; BATAOBA, S.; KUBOTA, Y.; KIKUCHI, M. Soy isoflavoneaglycones are absorbed faster and in high amounts than their glucosides in humans. Journal of Nutrition, v.130, p.1695-1699, 2000.

KAO, T. H.; CHEN, B. H. An improved method for determination of isoflavones in soybean powder by liquid chromatography, Chromatographia, v.56, p.423-430, 2002.

KLUMP, S. P. A.; MCDONALD, J. L.; BALLAM, J. M.; Determination of isoflavones in soy selected foods containing soyby extraction, saponification, and liquid chromatography: collaborative study, Journal of AOAC International,v.84, p. 1865-1883, 2001.

KNIGHT, D. C.; EDEN, J. A.A review of the clinical effects of phytoestrogens, Obstetric Gynecology, v. 87, p. 897-904, 1996. 
KURZER, M.S. Hormonal effects of soy isoflavones: studies in premenopausal and postmenopausal women. Journal of Nutrition,v. 130, p. 660-661, 2000 .

LEE, S. J.; YAN, W.; AHN, J.K.; CHUNG, I.M. Effects of year, site, genotype and their interactions on various soybeans isoflavones, Field Crops Research, n. 81, p. 181-192, 2003.

MILLER, J. C.; MILLER, J.N. Statistics and Chemometrics for Analytical Chemistry. 4. ed. Chichester: Ellis Horwood, 2005, 268 p.

MURPHY, P. A.; Separation of genistin, daidzin and their aglucones, and coumesterol by gradient highperformance liquid chromatography, Journal of ChromatographyA, v. 211, p.166-169,1981.

NAIN, M.; GESTETNER, B.; ZILKAH, S. Soybean isoflavones: characterization, determination, and antifungal activity. Journal of Agricultural Food Chemistry, v. 22, p. 806-810, 1974.

PASCUAL-TERESA, S.; HALLUND, J.; TALBOT, D.; SCHROOT, J.; WILLIANS, C.M.; BUGEL, S.; CASSIDY, A. Absorption of isoflavones in humans: effects of food matrix and processing. Journal of Nutritional Biochemistry, v. 17, p. 257-264, 2006.

RIBANI, M.; BOTTOLI, C.B.G.; COLLINS, C.H.; JARDIM, I.C.S.F.; MELO, L.F.C. Validação em métodos cromatográficos e eletroforéticos. Quimica Nova, v. 27, p. 771-780, 2004.

RIBANI, M.; COLLINS, C.H.; BOTTOLI, C.B.G. Validation of chromatographic methods: Evaluation of detection and quantification limits in the determination of impurities in omeprazole. Journal of Chromatography A, n. 1156, p. 201-205, 2007.

SETCHELL, D. R. K.; BROWN, N. M.; ZIMMERNECHEMIAS, L.; BRASHEAR, W.T.; WOLFE, B. E.; KIRSCHNER, A. S. Evidence for lack of absorption of soy isoflavone glycosides in humans, supporting the crucial role of intestinal metabolism for bioavailability. The American Journal of Clinical Nutrition, v. 76, p.447-453, 2001.

SEVERSON, R. K.; NOMURA, A.Y.M.; GROVE, J. S.; STEMMERMAN, G. N. A prospective study of demographics and prostate cancer among men of Japanese ancestry in Hawaii. Cancer Research, v. 49, p. 1857-1860, 1989.

SHAO, S.; DUNCAN, A.M.; YANG, R.; MARCONE, M. F.; RAJCAN, I.; TSAO, R. Systematic evaluation of pre-HPLC sample processing methods on total and individual isoflavones in soybeans and soy products. Food Research International, v.44, p.2425-2434, 2011.

SONG, T.; BARUA, K.; BUSEMAN, G.; MURPHY, P. A. Soy isoflavones analysis: quality control and a new internal standard. The America Journal of Clinical Nutrition, v. 68, p.1474-1479, 1998.

SOUCI, S.W.; FACHMANN, W.; KRAUT, H. Food Composition and Nutrition Tables. Stuttgart: Medpharm Scientific Publishers, 1994. 1182 p.

United States Pharmacopeia (USP) Convention. US Pharmacopeia 30: NF 25. Rockville, 2007.

WANG, H. J.; MURPHY, P. A. Isoflavone content in commercial soybean foods. Journal of Agricultural and Food Chemistry, v.42, p.1666-1673, 1994.

WATANABE, S.; KOESSEL, S. Colon cancer: an approach from molecular epidemiology. Journal of Epidemiology, v. 3, p. 47-61, 1993.

WISEMAN, H. C.; CLARKE, D. B.; BARNES, K. A.; BOWEY, E. Isoflavoneaglycone and glycoconjugate of high- and low-soy U.K. foods used in nutritional studies. Journal of Agricultural and Food Chemistry, v. 50, p.1404-1410, 2002.

YOUDEN, W.J.; STEINER E.H.The Association of Official Analytical Chemistry. Washington, DC, 1975, 88 p. 